Address for Correspondence: Dr. Xian-Ling Wang,

Department of Endocrinology, Chinese PLA General Hospital, Beijing 100853, China.

E-mail: wangxianling1972@sohu.com Deputy Chief Physician and Associate

Professor, $\mathrm{He}$ is a reviewer of 'Chinese Journal of Diabetes' and 'Chinese

Medical Journal', a member of the Study Group of Blood Glucose Monitoring of Chinese Diabetes Society. He has also participated in multiple National Natural Science Foundations and military research funds, obtained a second prize of Military Medical Achievement and published 8 papers in $\mathrm{SCl}$ journals with a cumulative impact factor of 18 .

\begin{tabular}{|l|}
\hline Access this article online \\
\hline Website: \\
www.intern-med.com \\
\hline DOI: \\
10.1515/jtim-2013-0005 \\
\hline Quick Response Code: \\
\hline \\
\\
\end{tabular}

\title{
Senile hypopituitarism: Characteristics of its diagnosis and treatment
}

\author{
Xian-Ling Wang, Ying-Qian Wang
}

Prof. Xian-Ling Wang, Department of Endocrinology, Chinese PLA General Hospital, Beijing 100853, China

\section{ABSTRACT}

Hypopituitarism is a clinical syndrome caused by reduced production of adenohypophyseal hormones. The clinical manifestations of senile hypopituitarism are more complicated and a delay in diagnosis and treatment may lead to rapid development of pituitary crisis. Therefore, clinicians should be alerted to senile hypopituitarism. For patients with hyponatremia, hypoglycemia and unexplained coma, an evaluation of pituitary function should be performed. Hormone replacement therapy for patients with confirmed hypopituitarism should be standardized and unauthorized dose reduction or withdrawal is not allowed.

Key words: diagnosis, hypopituitarism, the elderly, treatment

\section{INTRODUCTION}

As an extremely important endocrine organ in the human body, pituitary gland precisely controls the function of multiple endocrine glands. Pituitary gland can be subdivided into the adenohypophysis and the neurohypophysis. Hypopituitarism is a clinical syndrome resulting from reduced production of adenohypophyseal hormones. A trend of increase in the number of patients with hypopituitarism has been found in the elderly population. Compared with those of the young and adult patients with hypopituitarism, the clinical manifestations of senile patients are even more complicated and delay in diagnosis and treatment may lead to a rapid progression to life-threatening pituitary crisis. Attentions thus need to be paid on senile hypopituitarism clinically.

\section{ETIOLOGY AND PATHOGENESIS}

\section{Sheehan's syndrome}

Development of physiological hypertrophy and a very rich blood supply of the pituitary gland during pregnancy, childbirth bleeding, severe hypotension and shock could result in a significant reduction of blood supply to the hypophyseoportal system as well as massive ischemic necrosis of adenohypophysis, and thus lead to the rapid decline of gland function, namely Sheehan's syndrome..$^{[1]}$

\section{Hypothalamic and pituitary tumors}

Tumors can be classified as either functioning or non-functioning ones. Pituitary apoplexy induced by macroadenoma or adenoma hemorrhage could compress the normal pituitary tissue, resulting in a declined function of the normal pituitary tissue.

\section{Craniocerebral trauma, surgery or head and neck radiotherapy}

Craniocerebral trauma and damage to the hypothalamus or pituitary gland during surgery, especially pituitary stalk damage, usually cause a sudden decrease of blood supply to the pituitary gland. Pituitary tumors in situ or radiotherapy for metastatic cancer will also damage the function of the residual adenohypophysis. ${ }^{[2-4]}$

\section{Infection and lymphocytic hypophysitis}

The bacterial, fungal, viral infection as well as autoimmune inflammation in the hypothalamus or pituitary gland could cause damage to the adenohypophysis in different ways. Lymphocytic hypophysitis, an autoimmune inflammatory disease, has also been found recently in senile patients. 


\section{Pituitary apoplexy}

The rapid increase of tumor volume due to a sudden internal hemorrhage in pituitary macroadenoma will on one hand compress hypothalamus as well as other vital centers. On the other hand, it can compress normal pituitary tissue, leading to a rapid decrease of the gland function. It usually has an abrupt onset, with symptoms of headache, dizziness, vomiting and further a coma.

\section{Others}

Hematologic malignancies and malignant tumors can migrate to the pituitary gland and damage the function of adenohypophysis. Severe malnutrition due to a variety of causes can induce hypopituitarism. Some patients with idiopathic hypopituitarism could present only with pituitary involution and no obvious cause could be found.

\section{CLINICAL MANIFESTATIONS}

Hypopituitarism is mainly manifested by a series of syndromes, which are induced by functional decline in various target organs of the endocrine system. Adenohypophysis has strong compensatory ability, and the severity of decline in its function is related to the degree of pituitary damage.

\section{Functional decline in pituitary-gonadal axis}

Among adult patients, female patients mainly present with postpartum amenorrhea, agalactia, loss or disappearance of libido, and significant atrophy of mammary gland, ovary and uterus, while male patients have symptoms of degradation in secondary sexual characteristics, such as loss of underarm hair and pubic hair, erectile dysfunction and testicular atrophy. Postmenopausal women and men aged over 60 years seldom visit the hospital for the above symptoms and thus a delay in diagnosis and treatment often occurred.

\section{Functional decline in pituitary-thyroid axis}

It is manifested by chills, fatigue, anorexia and nausea; there could be paleness (associated with varying degrees of anemia), slight edema and dry skin, but generally no mucinous edema will be found; patients are found the lack of facial expressions and unresponsive and psychiatric symptoms could be developed in severe cases. The overall symptoms of hypometabolism are not as obvious as those of primary hypothyroidism (HT).

\section{Functional decline in pituitary-adrenal axis}

Patients present with fatigue and dizziness after standing for a long time; anorexia, nausea, vomiting; starvation intolerance and easy development of hypoglycemia; lower body resistance and easy development of upper respiratory tract infection. However, due to the less influence on secretion of aldosterone than on glucocorticoid, the severity of hyonatremia is not so obvious as it is found in primary adrenal cortical hypofunction. ${ }^{[5,6]}$

\section{Pituitary crisis}

For patients with hypopituitarism, a variety of stress conditions can induce pituitary crisis, such as infection, sepsis, diarrhea, vomiting, dehydration, long-time starvation or cold, cardiovascular and cerebrovascular accidents and insufficient preoperative preparation. Clinical manifestations include: (1) hyperpyrexia $>40^{\circ} \mathrm{C}$, which is rarely seen in the elderly; (2) hypothermia $<30^{\circ} \mathrm{C}$; (3) hypoglycemia; (4) hypotension; (5) overhydration; and (6) mixed type. All these types could be complicated by their corresponding clinical symptoms, which are manifested by the digestive, circulatory and neuropsychiatric symptoms, such as hyperpyrexia, circulatory failure, shock, nausea and vomiting, headache, psychiatric symptoms and even critical conditions including coma in severe cases.

\section{LABORATORY TESTS}

\section{Biochemical tests}

Results often show fasting hypoglycemia or low levels of blood glucose, low levels of serum sodium and serum chloride, while serum potassium is normal in most cases. The cause of hyponatremia and hypochloremia is associated with kidney drainage disturbance and increase of sodium consumption.

\section{Tests of endocrine function}

Tests of pituitary-gonadal axis function

Serum gonadotropins (luteinizing hormone [LH], folliclestimulating hormone [FSH]) are all elevated significantly in the normal elderly due to a decline in gonadal function (testis, ovary), whereas in senile patients with hypopituitarism, LH and FSH levels in serum are found decreased or at the lower limit of normal value, together with low levels of sex hormones (testosterone or estradiol). Results from the gonadotropin-releasing hormone stimulation test show extremely weak responses or negative responses of LH and FSH. Estradiol (female) or testosterone (male), which has significantly declined in senile patients, is considered useless for the differential diagnosis.

Tests of pituitary-thyroid axis function

The secondary HT induced by hypopituitarism is manifested by a significant decrease of total thyroxine $\left(\mathrm{TT}_{4}\right)$ and free thyroxine $\left(\mathrm{FT}_{4}\right)$, while the levels of total triiodothyronine $\left(\mathrm{TT}_{3}\right)$ and free triiodothyronine $\left(\mathrm{FT}_{3}\right)$ are not reduced significantly. More importantly, thyroidstimulating hormone (TSH) level is found inappropriately normal or slightly lower when there is an obvious decrease 
of $\mathrm{TT}_{4}$ and $\mathrm{FT}_{4}$, which is important in suggesting a functional decline in pituitary-thyroid axis.

Tests of pituitary-adrenocortical axis function

The levels of adrenocorticotropic hormone (ACTH) and free cortisol decline significantly at all-time points, with a low and flat curve for their secretion. The curves for ACTH and free cortisol are still found low and flat, or with slight responses in the hypoglycemia stimulation test.

\section{Growth hormone}

Basal growth hormone is normal or slightly lower. The curve for growth hormone secretion is still found at a low level in the hypoglycemia stimulation test.

\section{DIAGNOSIS AND DIFFERENTIAL DIAGNOSIS}

\section{Diagnosis}

Diagnosis of senile hypopituitarism is mainly based on the clinical manifestations of hypopituitarism, tests of endocrine function and relevant medical history or clinical signs. A further identification of pathogenesis and differential diagnosis is also needed after the confirmed diagnosis of hypopituitarism.

A previous medical history of childbirth bleeding and shock will facilitate the confirmed diagnosis of Sheehan's syndrome. Hypopituitarism induced by tumors usually has symptoms including enlargement of sella turcica and defect of visual field and pituitary magnetic resonance imaging examination will facilitate the further confirmation of pathogenesis. It will be easier to make a diagnosis in patients with a history of surgery or radiotherapy for hypothalamic/pituitary tumors. Attentions should be paid to the assessment of adenohypophysis function, when a low level of testosterone or estradiol is found in tests of gonadal hormone axis function without increases of LH and FSH levels.

\section{Differential diagnosis}

Clinical delay in diagnosis and treatment is usually due to attentions paid only to some prominent symptoms instead of taking account of the overall conditions for diagnosis. For every clinical patient with unexplained coma, especially those without a clear medical history, clinicians should be alerted highly to the possible occurrence of hypopituitarism. ${ }^{[7]}$

Primary adrenocortical insufficiency

Primary adrenocortical insufficiency has characteristic manifestations of typical skin and mucosal pigmentation, as well as more prominent hypotension and hyponatremia. Laboratory tests find a significant elevation of serum
ACTH level at all-time points, even higher than the measurable values.

\section{Primary HT}

The appearance of mucinous edema is more significant in primary HT. Measurement of plasma TSH levels will facilitate the differential diagnosis. TSH level is found increased in primary HT and normal or at a low level in the hypopituitarism. While there is an excessive TSH response in primary HT according to the thyroid releasing hormone (TRH) stimulation test, a small increase of TSH level is found in the hypopituitarism in response to TRH.

Autoimmune polyendocrine syndrome It is manifested by syndromes of primary multiple endocrine gland insufficiency. Due to lack of negative feedback inhibition of hormones affecting target endocrine organs, a significant increase is often found in levels of one or more hormones secreted by pituitary gland, such as ACTH, TSH, LH and FSH, which is considered important evidence for the differential diagnosis.

Chronic consumptive disease

Chronic consumptive disease could result in severe malnutrition of patients, with manifestations similar to hypopituitarism, such as weight loss, weakness and sexual hypofunction. The above symptoms could be gradually reversed after improvement of nutritional status and endocrine tests mainly show a low $\mathrm{T}_{3}-\mathrm{T}_{4}$ syndrome.

\section{TREATMENT}

The treatment of hypopituitarism can be classified as etiological treatment and symptomatic treatment. As to the etiological treatment, for treatment of hypopituitarism induced by space-occupying tumors, procedures including surgery, radiotherapy and chemotherapy could be selected according to the characteristics of tumors to release compression of the residual normal pituitary tissue due to the adjacent lesions. Hormones and/or other immunosuppressive agents are needed for treatment of hypopituitarism induced by lymphocytic hypophysitis to control the development and infiltration of inflammations.

\section{Hormone replacement therapy (HRT)}

The treatment of hypopituitarism is mainly aimed at supplementing hormones of the surrounding endocrine glands.

Adrenal cortical hormone

Oral prednisone at a daily dose of $2.5-7.5 \mathrm{mg}(5 \mathrm{mg}$ in the morning, $2.5 \mathrm{mg}$ in the afternoon) is generally administered for treatment of patients with steady-state of hypopituitarism. Improvement of the patients' physical and 
mental status as well as rapid improvement in symptoms of the hypoglycemia and hypotension can be found after treatment. The appropriate maintenance dose is determined based on the clinical indicators and a minimal dose that can maintain the normal blood pressure and serum sodium without development of central obesity will be enough. A double dose of prednisone will be needed for cases with stress conditions including hyperpyrexia, infection, surgery and trauma. Daily intravenous infusion of $100-300 \mathrm{mg}$ hydrocortisone can be performed when necessary, followed by a gradual reduction to the original maintenance dose after disappearance of the stress status. ${ }^{[6]}$

\section{Thyroid hormone}

Levothyroxine is most commonly used currently, beginning with a daily dose of $12.5-25 \mu \mathrm{g}$, followed by an increase of $25 \mu \mathrm{g}$ once every 2 weeks. A general dose of $75-100 \mu \mathrm{g}$ could be effective. After treatment, patients could be found with mitigation of chills, improvement of mental status and disappearance of edema. For senile patients complicated by multiple diseases, an initial dose of $12.5 \mu \mathrm{g}$ is used for safety reasons at the beginning of therapy, followed by an increase of $12.5 \mu \mathrm{g}$ once every 2 weeks. Monotherapy of thyroid hormone can aggravate symptoms of adrenal cortical insufficiency and thus the administration of thyroid hormone generally starts several days after adrenal cortical hormone therapy. A small number of senile patients have poor responses to the thyroid hormone therapy. Especially, the difficulties exist in supplementing with $\mathrm{TT}_{3}$ and $\mathrm{FT}_{3}$ to restore the normal level, which might be associated with the decreased conversion by 5 '-deiodinases in peripheral tissues of the elderly. After excluding the existence of other chronic diseases that contribute to the low $\mathrm{T}_{3}$ syndrome, thyroid hormone could be used in combination with a certain dose $(20-40 \mathrm{mg} /$ day $)$ of thyroid tablets $\left(\mathrm{T}_{3}\right.$ accounting for $1 / 3)$.

\section{Gonadal hormone}

Gonadal HRT is generally not necessary for senile patients.

\section{Growth hormone}

Growth hormone therapy is not needed for senile patients with hypopituitarism under physiological conditions. Some studies have suggested that whether the growth HRT has beneficial effects in the elderly still remains a question. ${ }^{[8]}$ However, short-term adjuvant therapy of growth hormone (injection) aiming at synergistically treating infection or promoting protein synthesis could achieve excellent therapeutic effects in cases complicated by severe infection or severe hypoproteinemia.

\section{Treatment of pituitary crisis}

After confirmed diagnosis of pituitary crisis, intravenous injection of $50 \%$ glucose solution $(40-60 \mathrm{~mL})$ should be performed first to correct hypoglycemia, followed by supplementation of $10 \%$ glucose saline. Hydrocortisone (50-200 $\mathrm{mg}$, dependent on the severity) is administered intravenously at the same time once every $6 \mathrm{~h}$ to rapidly improve the symptoms. Treatment of patients with circulatory failure needs to follow the therapeutic principle of shock, which requires supplementation of sufficient fluid volume as well as glucose and preparations of sodium chloride and for those with low levels of serum potassium; potassium chloride should be supplemented intravenously at the same time. Anti-infective therapy is needed in patients with infection. Diuresis should be promoted in patients with overhydration and attentions should be paid to the control of infused fluid volume to maintain the negative daily water balance of no more than $1 \mathrm{~L}$. In addition, basic nursing and expectant treatment also need to be reinforced to correct hypothermia or hyperpyrexia.

\section{CHARACTERISTICS OF SENILE HYPOPITUITARISM AND PRECAUTIONS IN THE TREATMENT}

The clinical manifestations of senile patients with hypopituitarism are usually atypical and diverse and some patients even fall into coma directly, easily resulting in the misdiagnosis and mistreatment clinically. For treatment of senile patients, special attentions are needed on the following points.

\section{Attentions to tests of adenohypophysis function in patients with hyonatremia}

Some elderly patients do not have obvious clinical symptoms of hypopituitarism, yet persistent low levels of serum sodium and serum chloride could be found by biochemical tests. A possible hypopituitarism needs to be considered after excluding the inducing factors such as insufficient intake, diuretic medication, etc. $^{[5]}$

\section{Attentions to identifying the causes of hypoglycemia}

Some elderly patients develop recurrent episodes of hypoglycemia without obvious causes, even a hypoglycemic coma in severe cases. A possible hypopituitarism also needs to be considered after ruling out the conditions including misuse of antidiabetic drugs, insulinoma, etc.

\section{Attentions to tests of adenohypophysis function in coma patients \\ Some senile patients often visit hospitals for unexplained coma. In cases with an unclear medical history, attentions are needed for simple screening of biochemical indicators including serum sodium, blood glucose, etc. A strong suspicion of possible pituitary crisis is needed for patients}


with complications of hyponatremia, hypochloremia and hypoglycemia. Venous blood could be collected first to evaluate the adenohypophysis function before emergency treatment and then a tentative intravenous infusion of hydrocortisone $(50-100 \mathrm{mg})$ is performed immediately, followed by observation of the therapeutic response. Meanwhile, the therapeutic program could be further adjusted as soon as the results of laboratory tests are available. Delay in rescue effort while waiting for test results is not allowed.

\section{Patients with hypopituitarism must strictly follow doctors' orders and unauthorized dose reduction or withdrawal is not allowed}

Senile patients with confirmed diagnosis of hypopituitarism must take their medication regularly during the HRT and avoid any unauthorized withdrawal. A dose increase is needed in accordance with doctors' orders especially under stress conditions such as pyrexia, diarrhea, etc. We often found patients making unauthorized withdrawal of glucocorticoid treatment due to fears of infection spreading in our clinical work, which led to a rapid development of pituitary crisis.

\section{REFERENCES}

1. KeleRENCES F. Sheehan's syndrome. Pituitary 2003;6:181-8.

2. Castro DG, Cecroar SA, Canteras MM. Radiosurgery for pituitary adenomas: Evaluation of its efficacy and safety. Radiat Oncol 2010;5:109.

3. Kokshoorn NE, Wassenaar MJ, Biermasz NR, Roelfsema F, Smit JW, Romijn JA, et al. Hypopituitarism following traumatic brain injury: Prevalence is affected by the use of different dynamic tests and different normal values. Eur J Endocrinol 2010;162:11-8.

4. El Chehadeh S, Bensignor C, de Monlde JV, MJV, M N, Huet F. The pituitary stalk interruption syndrome: Endocrine features and benefits of growth hormone therapy. Ann Endocrinol (Paris) 2010;71:102-10.

5. Petridis AK, Nabavi A, Doukas A, Buhl R, Mehdorn HM. Severe hyponatraemia in the setting of hypopituitarism associated with empty sella and herniation of the optic chiasm and gyrus rectus. J Clin Neurosci 2009;16:723-4.

6. Bouillon R. Acute adrenal insufficiency. Endocrinol Metab Clin North Am 2006;35:767-75, ix.

7. Foppiani L, Ruelle A, Bandelloni R, Quilici P, Del Monte P. Hypopituitarism in the elderly: Multifaceted clinical and biochemical presentation. Curr Aging Sci 2008;1:42-50.

8. Kokshoorn NE, Biermasz NR, Roelfsema F, Smit JW, Pereira AM, Romijn JA. GH replacement therapy in elderly GH-deficient patients: A systematic review. Eur J Endocrinol 2011;164:657-65.

How to cite this article: Wang $X L$, Wang $Y Q$. Senile hypopituitarism: Characteristics of its diagnosis and treatment. J Transl Intern Med 2013; 1(1): 13-7.

Source of Support: Nil, Conflict of Interest: None declared 